\title{
Reinventing solid state electronics: Harnessing quantum confinement in bismuth thin films
}

Farzan Gity, Lida Ansari, Martin Lanius, Peter Schüffelgen, Gregor Mussler, Detlev Grützmacher, and J. C. Greer

Citation: Appl. Phys. Lett. 110, 093111 (2017); doi: 10.1063/1.4977431

View online: http://dx.doi.org/10.1063/1.4977431

View Table of Contents: http://aip.scitation.org/toc/apl/110/9

Published by the American Institute of Physics

\section{Articles you may be interested in}

Magnetoresistance oscillations in MBE-grown Sb2Te3 thin films

Appl. Phys. Lett. 110, 092104092104 (2017); 10.1063/1.4977848

Solid solution strengthening in GaSb/GaAs: A mode to reduce the TD density through Be-doping Appl. Phys. Lett. 110, 092103092103 (2017); 10.1063/1.4977489

Elucidating gigahertz acoustic modulation of extraordinary optical transmission through a two-dimensional array of nano-holes

Appl. Phys. Lett. 110, 091910091910 (2017); 10.1063/1.4977430

Relative effect of edge versus basal plane functionalization on graphene/polymer interfacial thermal conductance - Role of in-plane phonons

Appl. Phys. Lett. 110, 093112093112 (2017); 10.1063/1.4977433

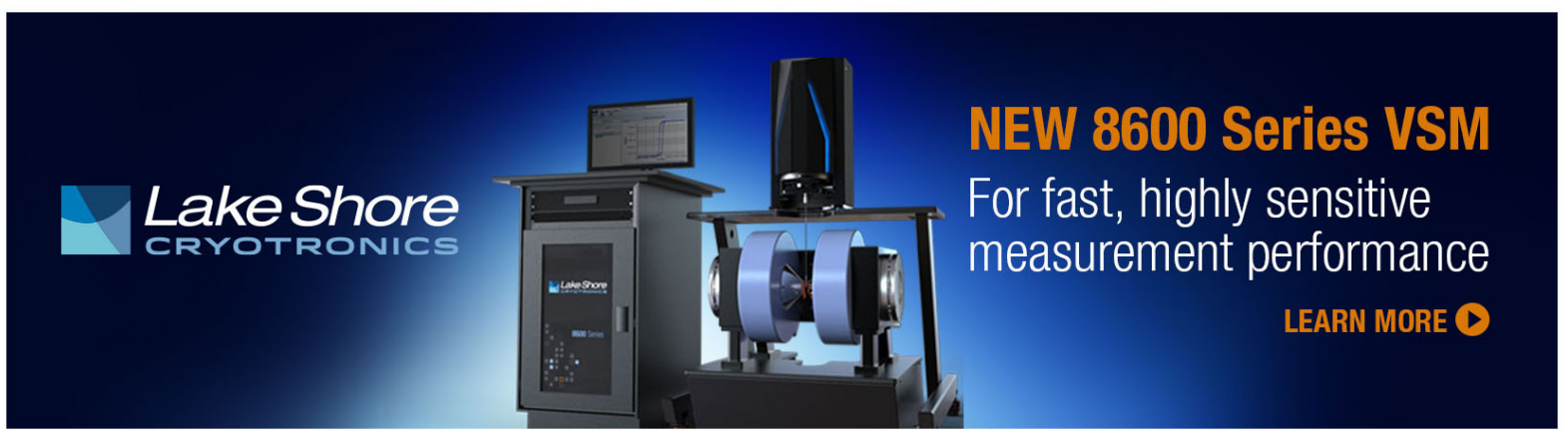




\title{
Reinventing solid state electronics: Harnessing quantum confinement in bismuth thin films
}

\author{
Farzan Gity, ${ }^{1}$ Lida Ansari, ${ }^{1}$ Martin Lanius, ${ }^{2}$ Peter Schüffelgen, ${ }^{2}$ Gregor Mussler, ${ }^{2}$ \\ Detlev Grützmacher, ${ }^{2}$ and J. C. Greer ${ }^{1, a)}$ \\ ${ }^{1}$ Tyndall National Institute, Lee Maltings, Dyke Parade, Cork T12 R5CP, Ireland \\ ${ }^{2}$ Peter Grünberg Institute 9 and Jülich Aachen Research Alliance (JARA-FIT), Research Center Jülich, \\ 52425 Jülich, Germany
}

(Received 22 December 2016; accepted 12 February 2017; published online 3 March 2017)

\begin{abstract}
Solid state electronics relies on the intentional introduction of impurity atoms or dopants into a semiconductor crystal and/or the formation of junctions between different materials (heterojunctions) to create rectifiers, potential barriers, and conducting pathways. With these building blocks, switching and amplification of electrical currents and voltages are achieved. As miniaturisation continues to ultra-scaled transistors with critical dimensions on the order of ten atomic lengths, the concept of doping to form junctions fails and forming heterojunctions becomes extremely difficult. Here, it is shown that it is not needed to introduce dopant atoms nor is a heterojunction required to achieve the fundamental electronic function of current rectification. Ideal diode behavior or rectification is achieved solely by manipulation of quantum confinement using approximately $2 \mathrm{~nm}$ thick films consisting of a single atomic element, the semimetal bismuth. Crucially for nanoelectronics, this approach enables room temperature operation. Published by AIP Publishing.

[http://dx.doi.org/10.1063/1.4977431]
\end{abstract}

Dennard or linear scaling of transistors to reduce costs while enhancing performance, ${ }^{1}$ now known as the "happy scaling" era, came to an end during the mid-1990s for integrated circuit manufacture. Since that time, various technology boosters have been implemented to overcome what were incorrectly foreseen as insurmountable barriers. Technology boosters rely on the introduction of alternative material combinations to reduce quantum mechanical tunnelling in a transistor's "OFF" state, the intentional introduction of strain to enhance electron and hole mobilities to increase currents in the "ON" state, and the use of silicon-on-insulator and/or three dimensional (3D) finFET structures to reduce short channel effects. ${ }^{2}$ Enormous scientific effort and financial investments have been made to overcome the limitations associated with the physical behaviour of materials and transistor architectures to enable continued scaling. These efforts have allowed the steady cadence for electronics miniaturisation observed during the 1960s and as expressed by Moore's "law." These advances have led to transistor critical dimensions of less than $10 \mathrm{~nm}$, or as expressed in number of atoms, approximately 20 silicon atoms or less. At such small scales, fundamental physical principles such as diffusion, solidsolubility limits, and statistical fluctuations pose challenges to manufacture at the length scales that industry seeks for increased functionality at lower power to fuel the improved performance that consumers and industries have come to expect with a concomitant lowering of cost.

For "end-of-the-roadmap" transistors, the number of atoms can be on the order of a few hundred to thousand and the introduction of even a few dopant atoms introduces extremely high dopant atom concentrations. Ultra-sharp doping concentration gradients to form $p-n$ junctions as required

${ }^{\text {a)} E-m a i l: ~ J i m . G r e e r @ t y n d a l l . i e ~}$ in conventional transistor designs is difficult to achieve at such dimensions, and random effects due to the positions of dopant atoms suggest that such $p-n$ junctions cannot be routinely realised on the scale of few nanometers. The very concept of a $p$ - $n$ junction breaks down. ${ }^{4}$ An alternative path forward is to eliminate the need for doping nanostructures by exploiting quantum effects that only arise on the nanoscale. ${ }^{5}$ Semimetals may be thought of as semiconductors with either a "zero or negative" forbidden energy gap or a band gap. As a result, quantum confinement in low-dimensional (twodimensional (2D), 1D) semimetal materials leads to a semimetal-to-semiconductor transition as the physical size of a nanostructure becomes comparable to the Fermi wavelengths of electrons and holes. ${ }^{6}$ Bulk bismuth has a rhombohedral crystal structure, which can be expressed in terms of a hexagonal unit cell, and is a semimetal with band overlap between the valence and conduction bands of $38 \mathrm{meV}$ and $98 \mathrm{meV}$ at $2 \mathrm{~K}$ and $300 \mathrm{~K}$, respectively. ${ }^{7-10}$ Here, the quantum confinement effect is used to demonstrate that for (111) Bi thin films of thickness less than $6 \mathrm{~nm}$, a "positive" band gap $>100 \mathrm{meV}$ emerges allowing for the formation of a metal-semiconductor junction between a thick (semimetallic) and thin (semiconducting) region in a single film. A metal-semiconductor junction can be either Ohmic, behaving as a simple resistor or it can act as current rectifying diode; in the latter case, a Schottky barrier is said to have formed. The formation of a Schottky barrier with near ideal diode operation at room temperature is reported here for a single elemental film, namely, bismuth, engineered to have two regions of differing thicknesses to form a heterodimensional junction between a three-dimensional (3D) or bulk region and two-dimensional (2D) film or nanolayer.

A quantum confinement induced band gap in Bi nanowires was predicted based on the electronic structure of bulk 
Bi and including the effects of confinement. ${ }^{6}$ For trigonal oriented square nanowires, a zero band gap material for an approximately $52 \times 52 \mathrm{~nm}^{2}$ cross section is expected to be seen with a sharply increasing band gap for decreasing nanowire cross section. Bismuth nanowires were patterned with cross sections ${ }^{11}$ of $40 \times 30$ and $40 \times 50 \mathrm{~nm}^{2}$. Electrical measurements indicate that the wires were indeed below the semimetal-to-semiconductor critical dimension. Electrical conductivity changes of a few percent relative to the conductivity of the nanowires at $\mathrm{T}=270 \mathrm{~K}$ were consistent with a band gap of the order of $10 \mathrm{meV}$. Similar results have been shown for bismuth nanowires and thin films with critical dimensions of the order of $20 \mathrm{~nm}$ and larger. However, these reported band gaps are only of the order of a few tens of meV (Refs. 7 and 12) or lower or comparable to the thermal energy at room temperature and hence substantially below the limits required for nanoelectronic applications.

Single crystalline bismuth nanowires were synthesized by electrochemical deposition in an aluminium oxide template. ${ }^{13}$ Thicker regions of diameter of $70 \mathrm{~nm}$ were grown in contact with narrower regions of approximately $30 \mathrm{~nm}$ diameter. An asymmetry in the currents by a factor of $\sim 1.5$ is found at forward and reverse bias voltages of $\pm 1 \mathrm{~V}$, which is suggested that it could be analogous to a metal-degenerate semiconductor junction. However, the small asymmetry between forward and reverse bias is too small to be of use in nanoelectronic devices and the current voltage (IV) characteristic cannot be described even as an approximation by the diode equation. Here, we demonstrate for much smaller critical dimensions that near ideal diode behaviour can be accomplished at room temperature with a rectification ratio of $\sim 75$ at voltages of only $\pm 0.15 \mathrm{~V}$ when correcting for parasitic series resistance.

To demonstrate room temperature diode behaviour, thin Bi films were grown by molecular-beam epitaxy (MBE) on $\mathrm{Si}(111)$ wafers. Prior to the deposition, the Si substrates were chemically cleaned by the HF-last RCA procedure to remove the native oxide and passivate the surface with hydrogen. The substrates were subsequently heated in-situ to $700^{\circ} \mathrm{C}$ for $20 \mathrm{~min}$ to desorb the hydrogen atoms from the surface. The Bi material flux was generated by an effusion cell operated at a temperature of $550^{\circ} \mathrm{C}$, which yields a growth rate of $17 \mathrm{~nm} / \mathrm{h}$. Thickness and crystallographic orientation are the key parameters determining the electronic structure of the thin films. $\mathrm{X}$-ray reflectivity (XRR) measurement for three exemplary $\mathrm{Bi}$ layers with thicknesses $28 \mathrm{~nm}, 14 \mathrm{~nm}$, and $5.7 \mathrm{~nm}$ can be seen in supplementary material (Fig. S1(A)), and supplementary material (Fig. S1(B)) shows X-ray diffraction (XRD) curves for the three films. The XRR curves display a high number of thickness oscillations evidencing smooth surfaces and interfaces with a simulated RMS value $<0.5 \mathrm{~nm}$. X-ray diffraction (XRD) for the same three layers reveals signatures at $2 \theta=22.49^{\circ}$ and $45.92^{\circ}$, which refer to the (111) and (222) reflections of $\mathrm{Bi}$ and hence indicate single crystal growth with the (111) orientation in the growth direction.

Hall measurements were performed to determine charge carrier mobility, carrier charge, free carrier concentrations, as well as resistivity. Temperature dependence of the carrier concentration is shown in Fig. 1 for the three Bi film thicknesses of $28 \mathrm{~nm}, 5.7 \mathrm{~nm}$, and $3.2 \mathrm{~nm}$. For the thicker Bi film, the carrier concentration is largely independent of temperature for
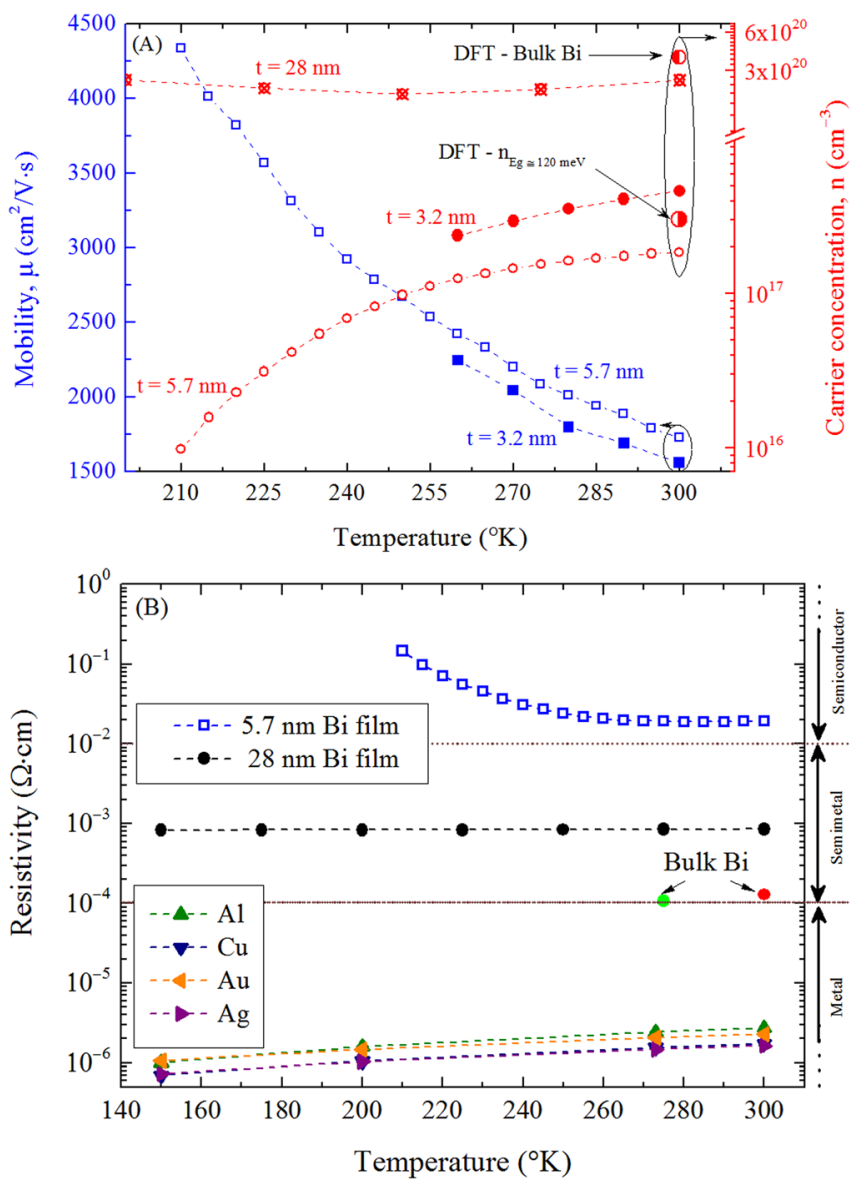

FIG. 1. Electrical characterization data for thin bismuth films of thicknesses $28 \mathrm{~nm}, 5.7 \mathrm{~nm}$, and $3.2 \mathrm{~nm}$. The thicker films are crystalline with (111) surface orientation, and the $3.2 \mathrm{~nm}$ film is polycrystalline. (a) Temperaturedependent mobility of the 5.7 and $3.2 \mathrm{~nm}$ Bi [111] films (blue). Carrier concentration as a function of temperature for Bi films with thicknesses 28, 5.7, and $3.2 \mathrm{~nm}$ reveals limited variation with temperature for the thicker $28 \mathrm{~nm}$ film but considerable reduction with temperature for the thinner 5.7 and $3.2 \mathrm{~nm}$ films. Carrier concentration estimates based on DFT band structures for bulk Bi and a thin film with a band gap $\approx 120 \mathrm{meV}$ are shown for comparison. Due to the larger quantum confinement effect in the $3.2 \mathrm{~nm}$ Bi film, the metal contact/Bi interface becomes rectifying at low temperatures hampering the electrical extraction for temperatures less than $260 \mathrm{~K}$. The same electrical limitation is observed for the $5.7 \mathrm{~nm}$ Bi film for temperatures less than $210 \mathrm{~K}$. (b) Resistivity of Bi films versus temperature is shown for the $5.7 \mathrm{~nm}$ and $28 \mathrm{~nm}$ Bi films, respectively. Resistivity for bulk Bi at two temperatures as taken from the literature ${ }^{14,15}$ and for several common metals ${ }^{15}$ are shown for reference.

the range shown indicating semimetallic behavior. However for the $5.7 \mathrm{~nm}$ film, more than an order of magnitude increase in the carrier concentration is seen over the temperature range from $200 \mathrm{~K}$ to $300 \mathrm{~K}$ revealing characteristic semiconducting behavior consistent with the onset of a quantum confinement induced band gap. Although the $3.2 \mathrm{~nm}$ Bi film is not single crystal (note the higher carrier concentration relative to the $5.7 \mathrm{~nm}$ film), it possesses a high mobility similar to the crystalline $5.7 \mathrm{~nm}$ film. The Hall mobility for both of the thinner films remains remarkably high as shown in Fig. 1(a). The estimated free carrier concentrations obtained from density functional theory (DFT) calculations for bulk Bi and a thin film with an induced band gap of $120 \mathrm{meV}$ are also shown in Fig. 1(a), which is in strong agreement with the experimental data. The temperature dependence of the resistivity for Bi thin films with varying thicknesses is shown in Fig. 1(b). Considering 
conventional resistivity classification of metals, semimetals, and semiconductors, the resistivity of the crystalline ultra-thin $5.7 \mathrm{~nm}$ Bi film is well within the semiconducting range and displays increased resistivity with lowering temperature.

The effects of quantum confinement and band folding determine the value of the confinement induced band gap in the (111)-oriented thin films, and electronic structure calculations were performed to correlate the theoretical predictions with the band gaps extracted from the electrical measurements. Standard theoretical methods for calculating band structures based on the density functional theory (DFT) are well known to suffer deficiencies with respect to predicting band gaps primarily due to approximations for the electronic exchange and correlation energies. These approximations typically result in a large and systematic underestimation of band gaps in semiconductors. The semimetallic band structure of bulk $\mathrm{Bi}$ found using DFT predicts a much larger band overlap of $\sim 160 \mathrm{meV}$ (Refs. 14 and 15) compared to the experimental value. To provide an improved description of the confinement effect in bismuth thin films, particularly with respect to determining band gap energies, the $G W(G$ : Green's function and $W$ : screened Coulomb interaction) method in conjunction with a many body perturbation theory (MBPT) correction is used. ${ }^{16}$ The $G W$ electron self-energy is used to correct the single particle energies resulting from the Kohn-Sham equations used to find the electron charge density within DFT. Due to the computational demands of $G W$ calculations, this approach was applied to films between approximately $1 \mathrm{~nm}$ and $2 \mathrm{~nm}$ thickness and used to extrapolate the effect of quantum confinement for thicker films. The role of surface chemistry in the band gap is investigated by considering $-\mathrm{H}$ and $-\mathrm{OH}$ terminated surfaces. In the DFT calculations without surface terminating species, the band gap disappears in films thicker than 2 bilayers due to the presence of surface states. However, removing surface states by hydroxyl and hydrogen terminations, the density of states (DoS) vanishes at the Fermi level in thin films with a band gap opening for thicknesses of several nanometers. As can be seen in Fig. 2, hydroxyl termination provides a slightly larger value of the band gap in comparison with hydrogen termination, an effect also found for silicon nanowires. ${ }^{17,18}$ The experimental samples have a native oxide layer on the Bi surfaces; therefore, the $-\mathrm{OH}$ termination better describes the surface chemistry and surface charge exchange in the films. The band gap extracted from the electrical measurements of the $\mathrm{Bi}$ film with a $5.7 \mathrm{~nm}$ thickness indicates a band gap of $125 \mathrm{meV}$, which is in good agreement with the theoretical predicted value of $117 \mathrm{meV}$ as extracted from the thermal activation of free carriers and assuming that the semiconducting Bi region is intrinsic. Hence, the electrical measurements are consistent with the theoretically predicted band gaps for (111)-oriented bismuth films with thickness $<6 \mathrm{~nm}$.

To form a heterodimensional rectifier by forming a three dimensional (3D) to two dimensional (2D) junction in bismuth nanolayers, an etch recipe was developed to selectively thin regions of the Bi film in a controlled manner. Ti/Au was deposited on a $12 \mathrm{~nm}$ semimetallic Bi film and patterned to form circular transfer length method (cTLM) electrical contacts. The metal contact structure was then used as a hard mask with the native oxide removed and the Bi film thinned using an argon plasma etch. Thinning of the exposed bismuth

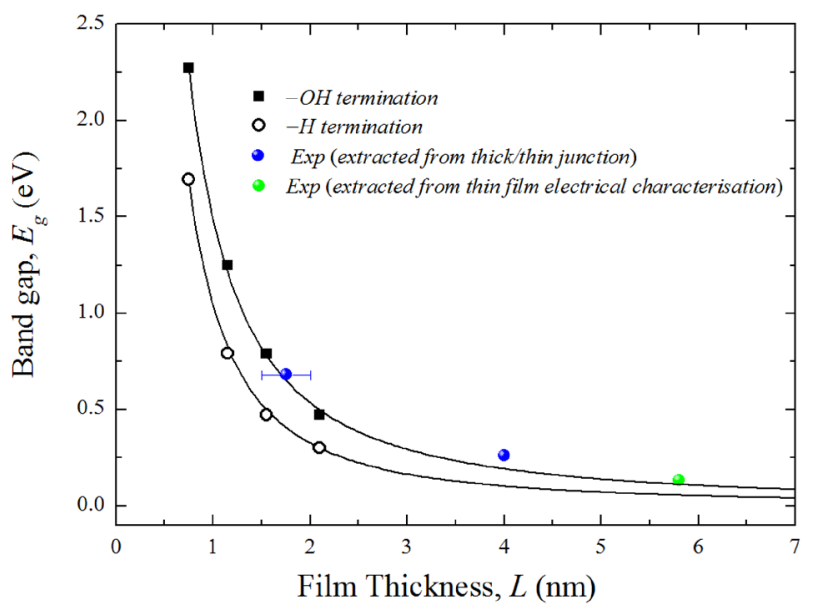

FIG. 2. Band gap versus film thickness and surface termination from $G W$ calculations for Bi (111) slabs. The solid curves show a power law fit $\alpha / L^{\beta}$ where $L$ is the film thickness and $\alpha$ and $\beta$ are fitting parameters. The green sphere indicates experimental data $\left(\mathrm{E}_{\mathrm{g}}=125 \mathrm{meV}\right)$. Blue spheres are experimental estimates based on extraction from the current-voltage characteristics at different temperatures for the thick/thin junctions.

was followed by evaporation of a $15 \mathrm{~nm} \mathrm{HfO}_{2}$ layer. This process was followed by an etch step to open the contact regions to allow for electrical measurement. Electrical measurements of the cTLM contacts on the $12 \mathrm{~nm}$ un-etched sample (reference sample) showed Ohmic current-voltage (IV) characteristics over a temperature range of $-40^{\circ} \mathrm{C}$ to $20^{\circ} \mathrm{C}$ as shown in the supplementary material yielding a contact resistance of $9 \times 10^{-4} \Omega \mathrm{cm}^{2}$. The resistivity obtained from the cTLM analysis of $\rho_{\mathrm{cTLM}} \approx 8 \times 10^{-4} \Omega \mathrm{cm}$ is in good agreement with the Hall measurements $\rho_{\text {cTLM }} \approx 6 \times 10^{-4} \Omega \mathrm{cm}$. To vary the thickness of the bismuth film exposed to the etch step, the etch duration was varied between $60 \mathrm{~s}$ and $80 \mathrm{~s}$ (Fig. 3). Electrical characteristics of the thick/thin devices are shown in Fig. 4 for the resulting $8.2 \mathrm{~nm}, 4 \mathrm{~nm}$, and $1.5 \mathrm{~nm}$ Bi films exposed to the etch step with thickness determined by TEM. The IV characteristic of the $12 \mathrm{~nm} / 8.2 \mathrm{~nm}$ junction is

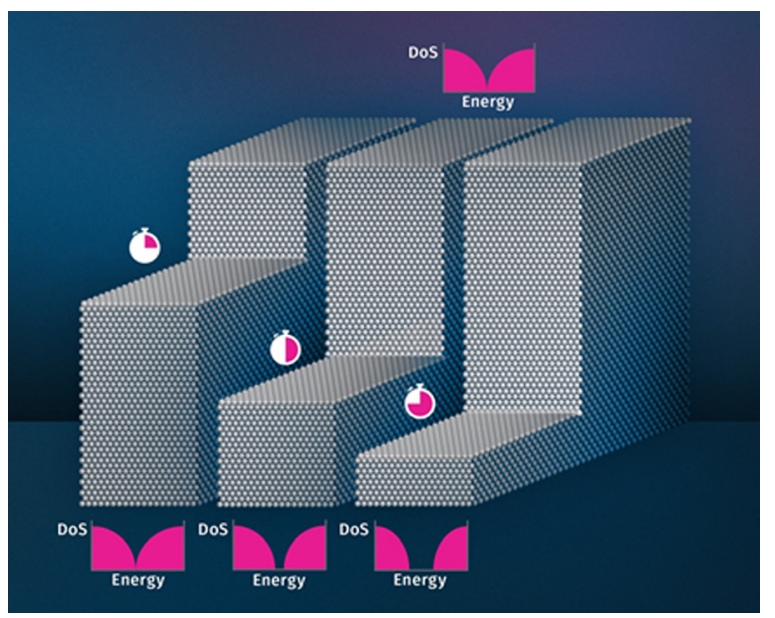

FIG. 3. Illustration of the "thick/thin" junctions formed with different etching times used to create a thin region. The stopwatch icon represents the proportional time for the thin region etch step. As the etched region is "thinned" a forbidden gap between the conduction and valence bands as shown in the schematic representation of the density of states (DoS) corresponding to the film thickness at the top and bottom of the figure emerges; detailed DoS plots for the film thicknesses considered are shown in the supplementary material. 
approximately temperature independent consistent with the quantum confinement effect not playing an appreciable role in $8.2 \mathrm{~nm}$ thick films. The dependence on temperature increases for the $12 \mathrm{~nm} / 4 \mathrm{~nm}$ junction since the band offset at the thick/ thin interface (i.e., $132 \mathrm{meV}$ ) is not large enough to prevent thermionic emission dominating the reverse current at room
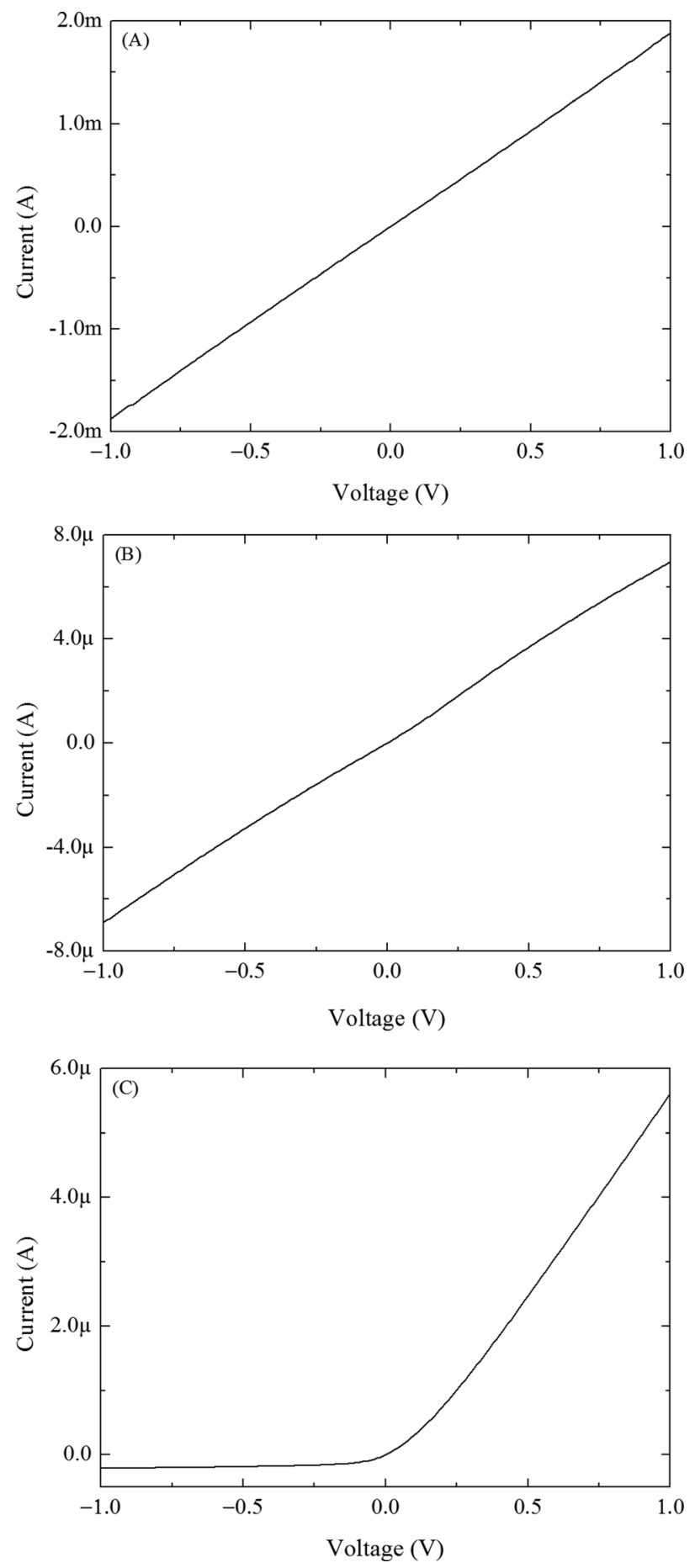

FIG. 4. Experimental current-voltage (IV) characteristics at $\mathrm{T}=20^{\circ} \mathrm{C}$ for the junction with thicknesses (a) $8.2 \mathrm{~nm}$, (b) $4 \mathrm{~nm}$, and (c) $1.5 \mathrm{~nm}$ in the thin region. Ohmic or near Ohmic behaviour is observed for the two thicker films. For the thick/thin junction in (c), diode behavior at room temperature is seen. As shown in the supplementary material, the IV characteristic when corrected for series resistance is consistent with exponential behavior in the forward direction. The $12 \mathrm{~nm} / 4 \mathrm{~nm}$ structure (b) becomes rectifying at $\mathrm{T}=-40^{\circ} \mathrm{C}$ as shown in the supplementary material consistent with smaller voltage barrier arising from the smaller band gap in the thin region with respect to the structure in (c). temperature. As the exposed bismuth region is further thinned to approximately $1.5-2 \mathrm{~nm}$, the formation of a Schottky barrier diode is observed through the rectifying nature of the IV characteristic. The semimetal-semiconductor barrier is further increased to $\sim 0.34 \mathrm{eV}$ for the $1.5 \mathrm{~nm}$ film as determined from the temperature dependence of the reverse saturation current. For a midgap Fermi level alignment, the estimated band gap is $\sim 0.70 \mathrm{eV}$, which is again in good agreement with the theoretical calculations as compared in Fig. 2. Near ideal diode behavior was observed for a diode fabricated in a heterodimensional junction using a single material, in this case bismuth. The current rectification is achieved at room temperature and with the observed asymmetry in the IV characteristic comparable to macroscale diode junctions (Fig. 4).

This study presents a key step forward toward a fundamental shift in nanoelectronics by exploiting quantum effects to mimic the operation of macroscopic microelectronics. It is revolutionary in the sense it relies on a material not currently used in nanoelectronics, yet this approach is capable of slotting into the highly developed machinery of integrated circuit manufacturing. The results prove that it is unnecessary to introduce dopant atoms to create a $p n$ junction nor is it required to form a heterojunction to achieve the fundamental function of current rectification. This work demonstrates that the fundamental laws of quantum mechanics can be harnessed to enable nanoelectronics innovation for electronic devices to be manufactured on the length scale of tens of atoms, and crucially, this can be achieved for room temperature device operation.

See supplementary material for (i) details of the materials growth and preparation; (ii) computational details that underpin theoretical results presented in the manuscript; and (iii) electrical characterization data that extend and support the experimental results presented in the manuscript.

This work has been funded by Science Foundation Ireland through the Principal Investigator Award No. 13/IA/ 1956. Calculations were performed on the high performance computing clusters provided at Tyndall and the Irish Centre for High End Computation (ICHEC).We thank Dr. Michael Schmidt at Tyndall for transmission microscopy imaging.

${ }^{1}$ R. H. Dennard, F. H. Gaensslen, L. Kuhn, and H. N. Yu, IEEE J. SolidState Circuits 9, 256-268 (1974).

${ }^{2}$ J. P. Colinge and J. C. Greer, Nanowire Transistors: Physics of Materials and Devices in One Dimension (Cambridge University Press, Cambridge, UK, 2016).

${ }^{3}$ G. E. Moore, Electronics 38, 114-117 (1965).

${ }^{4}$ J. P. Colinge, C. W. Lee, A. Afzalian, N. Dehdashti Akhavan, R. Yan, I. Ferain, P. Razavi, B. O’Neill1, A. Blake, M. White, A. M. Kelleher, B. McCarthy, and R. Murphy, Nat. Nanotechnol. 5, 225-229 (2010).

${ }^{5}$ L. Ansari, F. Fagas, J. P. Colinge, and J. C. Greer, Nano Lett. 12, 2222-2227 (2012).

${ }^{6}$ X. Sun, Z. Zhang, and M. S. Dresselhaus, Appl. Phys. Lett. 74, 4005-4007 (1999).

${ }^{7}$ S. Lee, J. Ham, K. Jeon, J. S. Noh, and W. Lee, Nanotechnology 21, 405701 (2010).

${ }^{8}$ A. J. Levin, M. R. Black, and M. S. Dresselhaus, Phys. Rev. B 79, 165117 (2009).

${ }^{9}$ J. Kim, W. Shim, and W. Lee, J. Mater. Chem. C 3, 11999-12013 (2015). 
${ }^{10}$ R. T. Isaacson and G. A. Williams, Phys. Rev. 185, 682-688 (1969).

${ }^{11}$ D. S. Choi, A. A. Balandin, M. S. Leung, G. W. Stupian, N. Presser, S. W. Chung, J. R. Heath, A. Khitun, and K. L. Wang, Appl. Phys. Lett. 89, 141503 (2006).

${ }^{12}$ C. A. Hoffman, J. R. Meyer, F. J. Bartoli, A. Di Venere, X. J. Yi, C. L. Hou, H. C. Wang, J. B. Ketterson, and G. K. Wong, Phys. Rev. B 48, 11431-11434 (1993).

${ }^{13}$ Y. T. Tian, G. M. Meng, G. Z. Wang, F. Phillipp, S. H. Sun, and L. D. Zhang, Nanotechnology 17, 1041-1045 (2006).
${ }^{14}$ A. B. Shick, J. B. Ketterson, D. L. Novikov, and A. J. Freeman, Phys. Rev. B 60, 15484-15487 (1999).

${ }^{15}$ I. Timrov, T. Kampfrath, J. Faure, N. Vast, C. R. Ast, C. Frischkorn, M. Wolf, P. Gava, and L. Perfetti, Phys. Rev. B 85, 155139 (2012).

${ }^{16}$ A. Marini, C. Hogan, M. Grüning, and D. Varsano, Comput. Phys. Commun. 180, 1392-1403 (2009).

${ }^{17}$ P. W. Leu, B. Shan, and K. J. Cho, Phys. Rev. B 73, 195320 (2006).

${ }^{18}$ M. Nolan, S. O'Callaghan, G. Fagas, J. C. Greer, and T. Frauenheim, Nano Lett. 7, 34-38 (2007). 